\title{
Direct-conversion switching-mode audio power amplifier with active capacitive voltage
} clamp

Ljusev, Petar; Andersen, Michael Andreas E.

Published in:

Power Electronics Specialists, 2005 IEEE 36th Conference on

Link to article, DOI:

10.1109/PESC.2005.1582037

Publication date:

2005

Document Version

Publisher's PDF, also known as Version of record

Link back to DTU Orbit

Citation (APA):

Ljusev, P., \& Andersen, M. A. E. (2005). Direct-conversion switching-mode audio power amplifier with active capacitive voltage clamp. In Power Electronics Specialists, 2005 IEEE 36th Conference on (pp. 2848-2854). IEEE. https://doi.org/10.1109/PESC.2005.1582037

\section{General rights}

Copyright and moral rights for the publications made accessible in the public portal are retained by the authors and/or other copyright owners and it is a condition of accessing publications that users recognise and abide by the legal requirements associated with these rights.

- Users may download and print one copy of any publication from the public portal for the purpose of private study or research.

- You may not further distribute the material or use it for any profit-making activity or commercial gain

- You may freely distribute the URL identifying the publication in the public portal 


\title{
Direct-conversion switching-mode audio power amplifier with active capacitive voltage clamp
}

\author{
Petar Ljušev, Michael A.E. Andersen \\ Ørsted • DTU Automation \\ Technical University of Denmark \\ Elektrovej DTU Building 325 \\ DK-2800 Kgs. Lyngby, Denmark \\ Email: pl@oersted.dtu.dk,ma@oersted.dtu.dk
}

\begin{abstract}
This paper discusses the advantages and problems when implementing direct energy conversion switching-mode audio power amplifiers. It is shown that the total integration of the power supply and Class $D$ audio power amplifier into one compact direct converter can simplify design, increase efficiency and integration level, reduce product volume and lower its cost. As an example, the principle of operation and the measurements made on a direct-conversion switching-mode audio power amplifier with active capacitive voltage clamp are presented.
\end{abstract}

\section{INTRODUCTION}

In the past few decades, the tremendous improvement of the power electronics components performance in terms of both speed and power capacity has opened some completely new power electronics application areas. The most challenging one is probably the audio power processing, which has been ruled by linear electronics since its very beginning. In audio amplification the switching-mode power electronics can ultimately prevail over the linear approach through its benefits like high efficiency, low losses, less volume and board space, leading to better product integration and lower price. When compared to the bulky linear audio amplifiers which can provide extraordinary low total harmonic distortion + noise $(\mathrm{THD}+\mathrm{N})$ levels and utilize simple control techniques to achieve it, the latter switching-mode approach (ex. Class D) is not without pitfalls either, especially with regard to its inevitable nonlinearity, introduction of time delays (rise, fall and dead times), need for output low-pass filter, switching frequency limiting the control bandwidth as well as the EMC problems. However, introduction of advanced control techniques [1] to compensate for the nonlinearities in the modulation and subsequent amplification phase has even led to several commercially available products, combining both high efficiency and high-end audio performance.

SIngle Conversion stage AMplifier (SICAM) [2],[3] represents an ultimate integration of a switching-mode power supply and a Class D audio power amplifier into a single unit capable of direct energy conversion from the single-phase AC mains. It is intended for the next generation of light, highly efficient and cheap audio appliances with satisfactory audio performance. It is also quite appealing for the Active Transducer (AT) [4] approach for direct conversion of the mains power into acoustic output in one simplified topological stage, like in the active loudspeaker systems and dedicated subwoofer units. By reshaping the audio conversion chain through dedication and integration of the power supply and the amplifier, the new direct topologies will multiplex the functions of the active switches and avoid redundant com- ponents to introduce much higher energy efficiency, even less volume and much better integration, yielding product price highly appealing for the cost driven segment of the market.

\section{DIRECT CONVERSION ISOLATED SWITCHING-MODE AUDIO POWER AMPLIFIERS}

The mainstream approach to building switching-mode audio power amplifiers, as shown in Fig. 1, is using a state-of-theart switching-mode isolated AC-DC power supply (SMPS) to provide stiff, intermediate DC bus voltage for powering a Class D switching-mode audio power amplifier.

This conventional approach is characterized by its modularity. In principle there is no need for communication between the power supply and audio power amplifier since they both have separate closed feedback control loops, therefore both parts can be manufactured separately, either in different departments of the same company or even in different companies without arising any incompatibility issues. Unfortunately, the aforementioned modular approach gives a false feeling of flexibility, since in the case of both the power supply and audio power amplifier working in switching-mode, some of the functions of the active switches in both modules can be integrated and some other components can be omitted. Increasing the level of dedication of the power supply and the audio power amplifier can even lead to completely new topologies, not foreseen before. These new structures referred as SICAMs are advantageous not only in terms of reduced component count due to avoidance of redundant components, but also the efficiency, power density, volume and cost can be improved.

While the total integration of the two separate modules in a single $\mathrm{AC}-\mathrm{AC}$ conversion stage can offer some obvious benefits, it introduces also few difficulties. Looking more thoroughly at the issue of single-phase direct conversion, the main problem that prohibits building the amplifier into a single stage without any power supply and energy storage components is

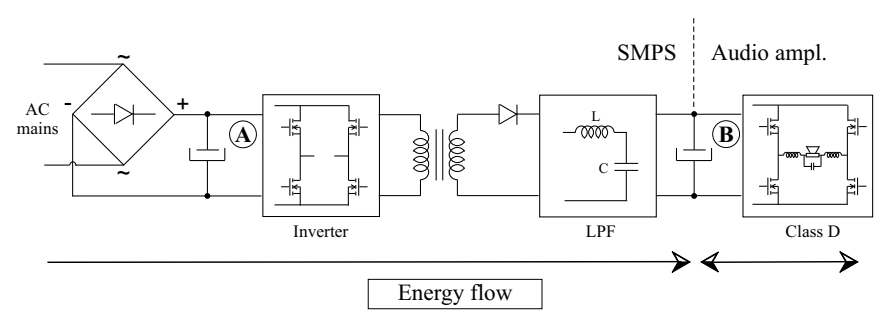

Fig. 1. Conventional switching-mode audio amplifier with a power supply 
the interrupted energy flow caused by the regular zero voltage crossings of the single phase voltage, twice per mains voltage period. While direct energy conversion without any reactive components (except for filtering purposes) in a three-phase matrix converter is possible due to the continuous or even constant energy flow provided by the system of three-phase $\mathrm{AC}$ voltages, single-phase matrix converter is completely inappropriate for the intended use due to its output voltage being phase-locked to the input voltage [5]. In other words, direct conversion audio power amplifier must include some kind of power supply just to provide energy storage and continuous power flow for the subsequent amplifying stage.

The conventional switching-mode audio power amplifier approach in Fig. 1 solves the problem of energy storage in elegant, but redundant and expensive way. The isolated AC-DC SMPS has two sets of bulky energy storage DC capacitors, marked with A and B in Fig. 1, placed on both sides of the isolation barrier, which is driven with $\mathrm{HF}$ AC voltage from the inverter to reduce the magnetics dimensions. These redundant capacitors essentially perform the same energy buffering function, but they are necessary for proper operation of the isolated AC-DC SMPS. The presence of secondary side rectifier prohibits the bidirectional power flow across the isolation barrier, so the low impedance capacitor B must be capable of buffering the energy returned from the reactive load without disturbing the SMPS output voltage too much. Finding a way to use only one energy storage capacitor at the mains input can lead to significant savings in power losses, board space, volume, component count and therefore overall cost. This however asks for redesign of the present solutions, by making the switching-mode audio power amplifier capable of directly operating with AC input voltage from the isolation transformer.

The proposed direct-conversion audio power amplification solution, referred herein as SICAM and shown in Fig. 2 is similar to the so called HF-link converter [6] and features closely interconnected SMPS - audio power amplifier topology that achieves multiplexing of the switchers function, has only a single energy storage capacitor A and provides longer bidirectional flow through the integrated audio power amplifier. However, this has its own difficulties, since the AC voltage present on the secondary side of the isolation transformer necessitates use of bidirectional switches in the audio power amplifier, slightly complicating its design. Actually, most of the peculiarities arise from the need to find a safe way to commutate the load current through the secondary side bidirectional bridge, since the diode freewheeling path during the blanking time intervals present in conventional Class D amplifiers is nonexistent here.

When compared to the conventional solution with separate SMPS and Class D amplifier, the proposed compact direct energy conversion switching-mode audio power amplifier i.e. SICAM results in numerous advantages like: simpler topologies, reduced parts count, no redundancy, increased efficiency, lower power losses, less heatsinking material, less board space, reduced product volume and subsequently lower cost.

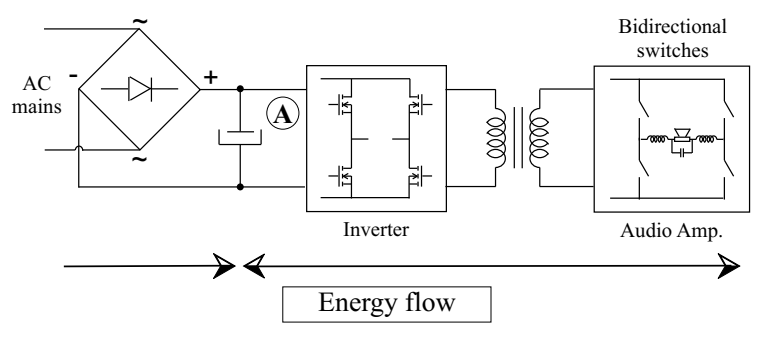

Fig. 2. The proposed isolated SICAM solution

\section{SICAM WITH ACTIVE CAPACITIVE VOLTAGE CLAMP}

\section{A. Topology and operation basics}

The SICAM with active capacitive voltage clamp, shown in Fig. 3, belongs to the group of direct conversion switchingmode audio power amplifiers which has non-modulated transformer voltages, like the previously presented MasterSlave operated SICAM [2], [3] and the Synchronized safecommutated SICAM [7]. This means that the inverter input stage before the insulation barrier of both aforementioned SICAMs is operated in a way that creates symmetrical, rectangular AC voltage with duty cycle of $50 \%$, while all audio modulation is undertaken on the secondary side. When compared to the solutions with phase-shifted PWM modulated transformer voltages [8], [9], using non-modulated transformer voltages leads to: easily predictable transformer flux levels, additional audio channels can reuse the same input stage and transformer, auxiliary biasing windings can be easily fitted on the same core, lower switching frequency on the primary side decreases switching and magnetics losses, no problems exist with transferring the audio reference over the isolation barrier to the primary side, no need for tightly interconnecting the control stages of the input and output stage and no need for 3-level modulation in the input stage. However, the latter solutions suffer from rather involved load current commutation from the outgoing to the incoming switches of the bidirectional bridge in the output stage, where the load current should not

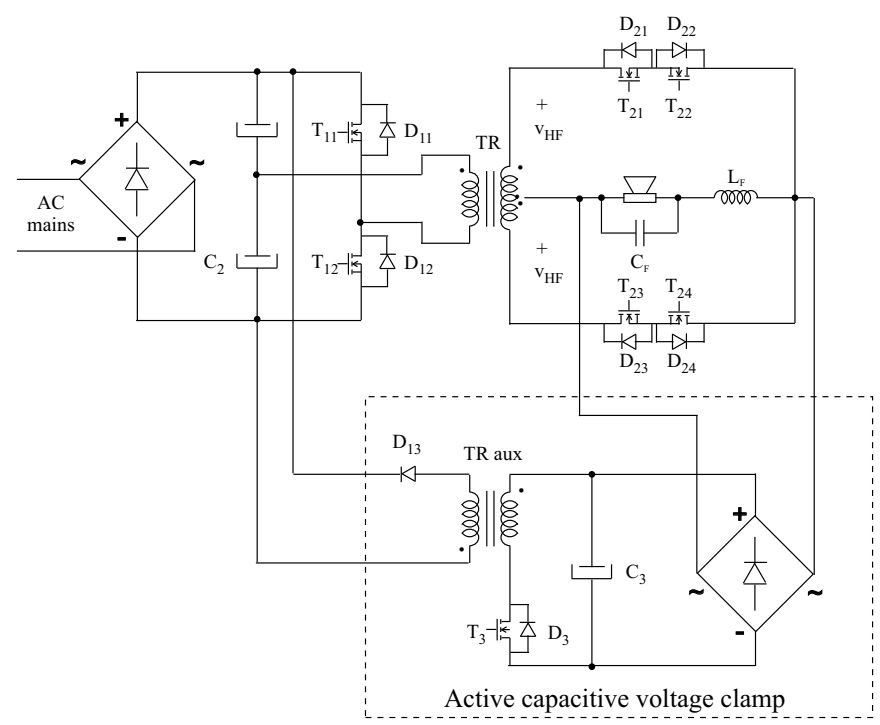

Fig. 3. Isolated SICAM with an active capacitive voltage clamp 
be interrupted and the HF-link i.e. the transformer should not be short-circuited at any instant.

Previously presented Master-Slave operated SICAM [2], [3] and Synchronized safe-commutated SICAM [7] introduced a new voltage-controlled switching pattern that alleviates load current commutation by choosing the right switching sequence of the incoming and the outgoing switches, depending on the HF-link voltage polarity. Its main drawback is the involved control strategy that prohibits switching both the input and the output stage simultaneously, while the intentionally inserted delay times for commutation purposes when advancing through the safe-commutation switching pattern give additional rise to output voltage distortion.

The proposed SICAM with active capacitive voltage clamp solves the commutation problem by allowing very short dead or blanking time $t_{b l}$ between the outgoing and incoming switches, and in the meantime clamping the output filter and load voltage by diverting the load current into the clamp capacitor $C_{3}$ through a very fast full-bridge rectifier. The clamp itself is called active, since the energy dumped in the clamp capacitor during the short dead time intervals of the secondary side switches is not dissipated, but is instead returned to the primary side with a simple, small, isolated single-switch auxiliary converter, shown in the lower right corner in Fig. 3 as a flyback converter. The auxiliary converter is operated in a way that regulates the clamp capacitor voltage $V_{c l}$ to a value that is slightly higher than the highest transformer secondary voltage expected during high voltage on the input line $V_{s, \max }$, so that the clamp capacitor is charged only during the dead time interval and not throughout the rest of the switching interval. Although the task looks almost the same as in a common flyback converter, the regulated quantity is not the output voltage but the voltage of the clamp capacitor. Therefore, this active clamping technique poses some new challenges in the design of the auxiliary converter.

Since the clamp is connected in parallel with the combination of only the output filter and the loudspeaker, it is very important to keep the transformer secondary side leakage inductance as low as possible, which will limit the ringing during the dead time intervals due to the stored magnetic energy to a minimum.

Although the particular implementation of the SICAM with active capacitive voltage clamp depicted in Fig. 3 features halfbridge inverter on the primary side and single-ended amplifier on the secondary side, the selection of input and output stage topologies is performed separately for each design case depending primarily on the input voltage and output power levels.

\section{B. PWM modulator and control of the SICAM}

The SICAM with active capacitive voltage clamp runs with a modified PWM modulator, shown in Fig. 4, where the double-sided triangular carrier has a constant carrier frequency $f_{c 2}$ and amplitude $V_{c}$, introducing constant gain $K_{P W M}=1 / V_{c}$ up to the half of the carrier frequency. The additional logical gates are used to obtain the correct gate signals, according to the instantaneous HF-link polarity, which changes with the switching frequency of the inverter stage $f_{s 1}$.

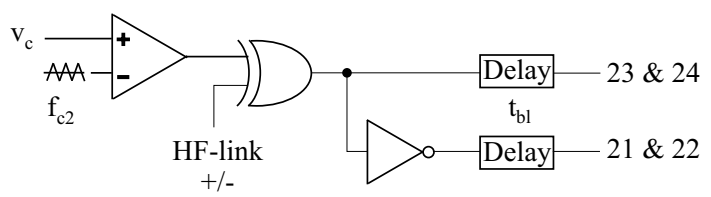

Fig. 4. Modified PWM modulator for SICAM

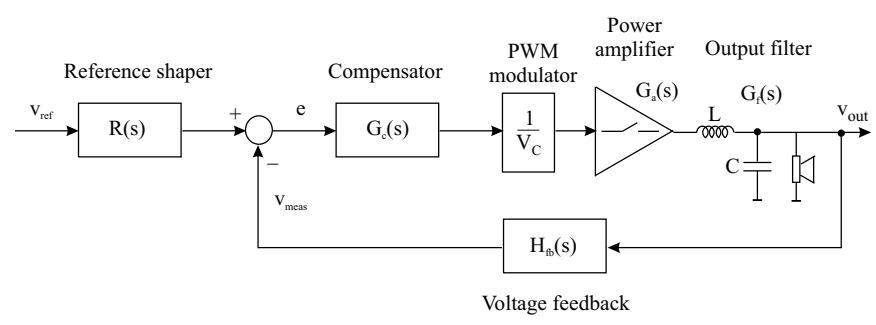

Fig. 5. Block diagram of simple output voltage control

Therefore, the actual switching frequency of the bidirectional bridge $f_{s 2}$ is:

$$
f_{s 2}=f_{s 1}+f_{c 2}
$$

which means that the switching frequency of the bidirectional bridge does not depend solely on the triangular carrier frequency, like in the Class D amplifiers, but also on the HF-link frequency. Modifications of the proposed PWM method in Fig. 4 are possible, which guarantee $f_{s 2}=f_{c 2}$ and therefore lower switching losses, but on behalf of slightly more complex modulator scheme [7].

The selection of control approach usually represents a tradeoff between simplicity and performance. The block diagram of simple output voltage control through single closed feedback loop is presented in Fig. 5. The presence of the output filter double poles makes it very difficult to achieve simultaneously proper phase margin and high control bandwidth i.e. the loop gain within the desired power bandwidth must be kept to reasonable values for robust and reliable operation. Other options are also possible, like cascade control with fast inner current loop and slower outer voltage loop [7] or even use of self-oscillating modulators [10].

\section{Clamp and auxiliary converter design}

When compared to similar dissipative solutions [11] where a properly chosen resistor is used to dissipate the energy dumped in the clamp capacitor and keep the clamp voltage from raising abnormally even at highest loading, the active clamp approach gives much higher efficiency. This is done, however, on expense of increased complexity and cost due to several additional power components, but the auxiliary converter itself processes only a small fraction of the total audio power amplifier power, so it is rather small and cheap to implement.

For comparison purposes, the power dissipated in a dissipative clamp resistor $R_{c l}$ is almost independent of the SICAM instantaneous output voltage, since the clamp capacitor is always charged through the clamp rectifier to at least the transformer secondary voltage:

$$
P_{d i s}=\frac{V_{s}^{2}}{R_{c l}}
$$


On the other hand, using an active load voltage clamp with auxiliary converter preserves and regenerates the charge dumped in the clamp capacitor. Assuming that sinusoidal load current with maximum value of $I_{o, \max }$ and variable modulation index $M$ is diverted into the clamp at the switching frequency of the bidirectional bridge $f_{s 2}$ and with duration equal to its blanking time $t_{b l}$, the average current into the clamp is:

$$
I_{c l, a v}=t_{b l} f_{s 2} \frac{2}{\pi} M I_{o, \max }
$$

Power handled by the auxiliary converter $P_{c l}$ of the active clamp is calculated as:

$$
P_{c l}=V_{c l} I_{c l, a v}=V_{c l} t_{b l} f_{s 2} \frac{2}{\pi} M I_{o, \max }
$$

and the active clamp power loss is just a fraction of $P_{c l}$ :

$$
P_{l o s s, c l}=\eta_{c l}\left(P_{c l}\right) \cdot P_{c l}
$$

The most important quantity for the design of the auxiliary converter is the maximum transferred charge $Q_{t r, \max }$ from the load to the clamp capacitor during one period of the auxiliary converter operation $T_{a u x}=1 / f_{\text {aux }}$, assuming that the latter is able to return the whole charge to the primary side and regain the balance on the clamp capacitor voltage. With maximum modulation index of one $M_{\max }=1$, maximum transferred charge becomes:

$$
Q_{t r, \max }=\frac{f_{s 2}}{f_{a u x}} I_{o, \max } t_{b l}
$$

The maximum charge $Q_{a u x, \max }$ that can be transferred from the clamp capacitor to the primary side with the discontinuous conduction mode (DCM) auxiliary converter is calculated by using its peak inductor current $I_{a u x, p k}$ and the maximum duty cycle $D_{\max }$ :

$$
Q_{a u x, \max }=\frac{1}{2} I_{a u x, p k} D_{\max } T_{a u x}=\frac{V_{c l} D_{\max }^{2}}{2 L f_{a u x}^{2}}
$$

which limits the maximum inductance $L_{\max }$ of the auxiliary converter operating in DCM needed to entirely regenerate the maximum dumped charge $Q_{t r, \max }$ from the clamp:

$$
L_{a u x, \max }=\frac{V_{c l} D_{\max }^{2}}{2 Q_{t r, \max } f_{a u x}^{2}}=\frac{V_{c l} D_{\max }^{2}}{2 f_{s 2} f_{a u x} I_{o, \max } t_{b l}}
$$

Other operating modes of the auxiliary converter are also possible, like combining continuous conduction mode CCM with large load currents and DCM with low load currents, but pure DCM operation leads to smallest size magnetics.

\section{Control of the active clamp auxiliary converter}

The control of the auxiliary converter represents a challenge for itself, due to the fact that the controlled quantity is the clamp capacitor voltage i.e. the source voltage, instead of the load voltage as with the conventional SMPS. The small-signal $\mathrm{AC}$ model of the aforementioned flyback auxiliary converter can be developed by using the modified buck-boost converter schematic in Fig. 6 and following the same principles like in [12].

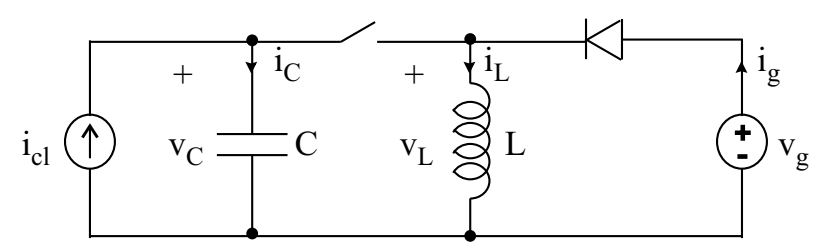

Fig. 6. Buck-boost auxiliary converter

It can be shown [13] that the CCM control-to-clamp-voltage transfer function $G_{v c d, c c m}$ obtained from the small-signal AC model of the buck-boost auxiliary converter in Fig. 6 is:

$$
G_{v c d, c c m}=\frac{v_{c}}{d}=\frac{D V_{g}\left(1+\frac{D^{\prime}}{D}\right)-s L \frac{I_{c l}}{D}}{D^{2}+s^{2} L C}
$$

and the DCM control-to-clamp-voltage transfer function $G_{v c d, d c m}$, as derived from the small-signal averaged switch model of the DCM buck-boost auxiliary converter in Fig. 7, is:

$$
\begin{aligned}
& G_{v c d, d c m}=\frac{v_{c}}{d}= \\
& =\frac{r_{1} j_{2}+f_{1}\left(1-h_{2}\right)+s L j_{2}}{\left(1-h_{2}\right)\left(1-k_{C}\right)-r_{1} g_{c}+s\left(r_{1}-L g_{C}\right)+s^{2} L C}
\end{aligned}
$$

where the coefficients in (10) are obtained by differentiating the input voltage $v_{1}$ and the output current $i_{2}$ of the averaged switch network:

$$
\begin{aligned}
& v_{1}=(1-d) v_{C}+d v_{g}-\frac{2 L i_{L} v_{g}}{d T_{s} v_{C}}=\gamma_{1}\left(v_{g}, v_{C}, i_{L}, d\right)= \\
& =k_{g} v_{g}+k_{C} v_{C}+r_{1} i_{L}+f_{1} d \\
& k_{g}=\left.\frac{\partial \gamma_{1}\left(v_{g}, V_{C}, I_{L}, D\right)}{\partial v_{g}}\right|_{v_{g}=V_{g}}=D-\frac{2 L I_{L}}{D T_{s} V_{C}} \\
& k_{C}=\left.\frac{\partial \gamma_{1}\left(V_{g}, v_{C}, I_{L}, D\right)}{\partial v_{C}}\right|_{v_{C}=V_{C}} ^{v_{g}=V_{g}}=(1-D)+\frac{2 L I_{L} V_{g}}{D T_{s} V_{C}^{2}} \\
& r_{1}=\left.\frac{\partial \gamma_{1}\left(V_{g}, V_{C}, i_{L}, D\right)}{\partial i_{L}}\right|_{i_{L}=I_{L}}=-\frac{2 L V_{g}}{D T_{s} V_{C}} \\
& f_{1}=\left.\frac{\partial \gamma_{1}\left(V_{g}, V_{C}, I_{L}, d\right)}{\partial d}\right|_{d=D}=-V_{C}+V_{g}+\frac{2 L I_{L} V_{g}}{D^{2} T_{s} V_{C}} \\
& i_{2}=i_{L}-\frac{d^{2} T_{s} v_{c}}{2 L}=\gamma_{2}\left(v_{C}, i_{L}, d\right)= \\
& =g_{C} v_{C}+h_{2} i_{L}+j_{2} d \\
& g_{C}=\left.\frac{\partial \gamma_{2}\left(v_{C}, I_{L}, D\right)}{\partial v_{C}}\right|_{v_{c}=V_{C}}=-\frac{D^{2} T_{s}}{2 L} \\
& h_{2}=\left.\frac{\partial \gamma_{2}\left(V_{C}, i_{L}, D\right)}{\partial i_{L}}\right|_{i_{L}=I_{L}} ^{v_{c}=V_{C}}=1 \\
& j_{2}=\left.\frac{\partial \gamma_{2}\left(V_{C}, I_{L}, d\right)}{\partial d}\right|_{d=D} ^{i_{L}=I_{L}}=-\frac{2 D T_{s} V_{C}}{2 L}
\end{aligned}
$$

The use of current-mode control simplifies the control synthesis by essentially removing the pole associated with the inductor dynamics and making the inductor current a controlled quantity. This is, however, more important with the CCM auxiliary flyback converter where the inductor pole 


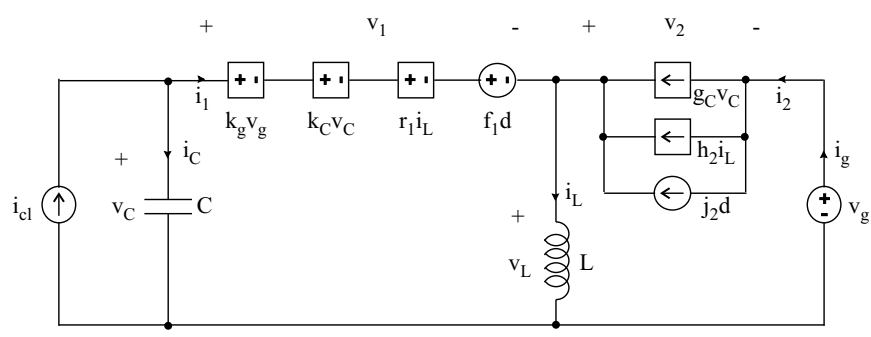

Fig. 7. Small-signal AC model of DCM buck-boost auxiliary converter

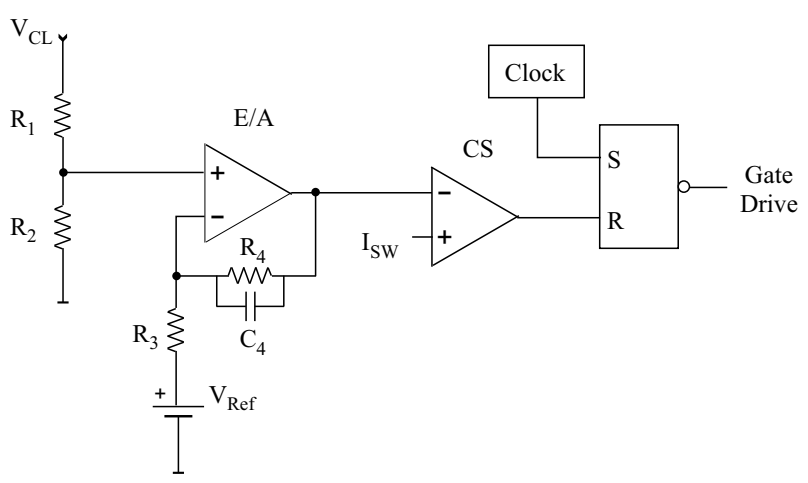

Fig. 8. Current-mode control of the auxiliary converter

appears at lower frequencies, than with the DCM auxiliary flyback converter, where the inductor pole is already shifted to high frequency comparable to the switching frequency.

From the practical viewpoint, increase in the clamp capacitor voltage should result in larger duty cycle as to regenerate more charge from the clamp, i.e. the controller should be built around a non-inverting amplifier. To alleviate the control design, current-mode control of the auxiliary converter was implemented on a standard SMPS control chip, like shown in Fig. 8, where both the inverting and non-inverting pin of the error amplifier are accessible to build a non-inverting compensator.

The implementation of the capacitive voltage clamp offers some unique benefits, which are not found elsewhere. The most prominent one is the increased safety against induced overvoltages in the case of loosing a secondary side gate-drive for bidirectional switches or other malfunctions that interrupt the usual load current path, which is functionally analogous to the three-phase matrix converter dissipative clamp. One other disputable advantage of the active capacitive voltage clamp is the marginally improved ride-through capability of the SICAM in the case where AC mains input voltage is decreased or even lost for a very short period of time, during which the auxiliary converter feeds some charge from the capacitor-dump to the primary side storage capacitor and supports the amplifier operation.

\section{MEASUREMENT RESUlTS ON THE PROTOTYPE}

In order to test the feasibility of the proposed approach, a $100 \mathrm{~W}$ prototype of the isolated SICAM with active capacitive voltage clamp was constructed, as shown in Fig. 9. The primary switching frequency was selected to be $f_{s 1}=100 \mathrm{kHz}$ to optimize the transformer weight vs. losses, while the secondary switching frequency was chosen to be $f_{s 2}=225 \mathrm{kHz}$,

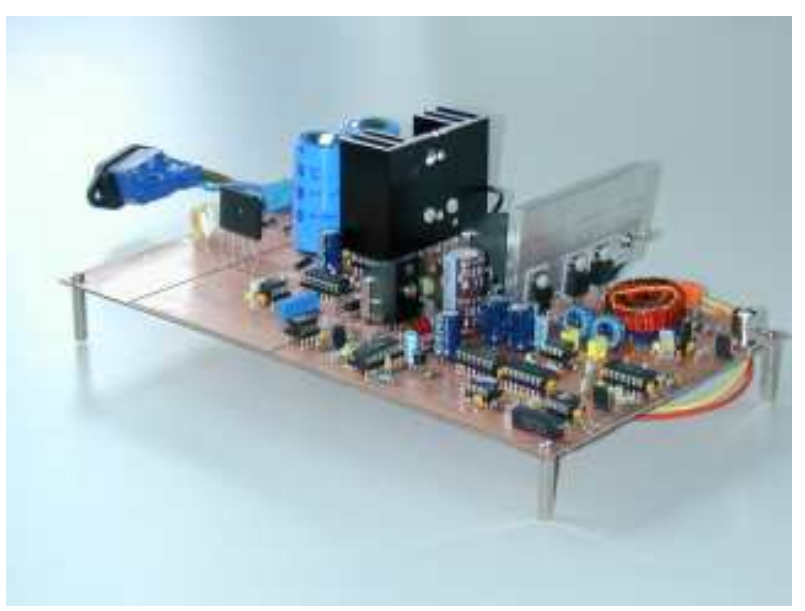

Fig. 9. Photo of the prototype SICAM with active capacitive voltage clamp

in order to avoid having too many primary-secondary intermodulation harmonics within the audio range. Independent primary and secondary switching frequencies lead to simpler design with much easier start-up circuitry and possibility to optimize the losses and dimensions of both the input stage and the transformer, but new designs will concentrate on frequency locking and synchronizing the operation of the input and output stage to improve the audio performance by avoiding intermodulation cross products.

Operation of the SICAM with active capacitive voltage clamp with more and less detail is shown in Fig. 10 and Fig. 11 respectively. Notice that the HF-link voltage has rectangular waveform with duty cycle of $50 \%$, while the bridge voltage applied across the combination of the output filter and loudspeaker is chopped from the HF-link voltage in a PWM manner.

The THD+N vs. frequency measurement in Fig. 12, THD+N vs. power measurement in Fig. 13, intermodulation distortion in Fig. 14 and FFT measurement in Fig. 15 show the promising audio performance of the prototype. The power efficiency of the prototype at maximum power of $100 \mathrm{~W}$ is around $80 \%$, but this is likely to be improved in the next more advanced designs.

\section{INTEGRATED MAGNETICS FOR SICAM WITH ACTIVE CAPACITIVE VOLTAGE CLAMP}

Although the proposed SICAM with active capacitive voltage clamp has much higher efficiency due to the recycling of the dumped clamp energy, it is by no means as simple and cost effective as the dissipative clamp solutions [11]. One way to reduce the complexity of the active clamp and reduce component count is to introduce special integrated magnetics, which incorporates the auxiliary transformer on the same magnetic core with the main power transformer. The principal approach is shown in Fig. 16, where the main transformer winding $N_{2}$ creates flux $\Phi_{2}$ in the center leg and outer windings, which is independent of the flux $\Phi_{1}=\Phi_{3}$ flowing entirely in the outer legs, caused by the magnetomotive forces of the serially connected and identical windings $N_{1}$ and $N_{3}$. In this way, the magnetic structure associated with the central leg and winding $N_{2}$ is totally independent from the 


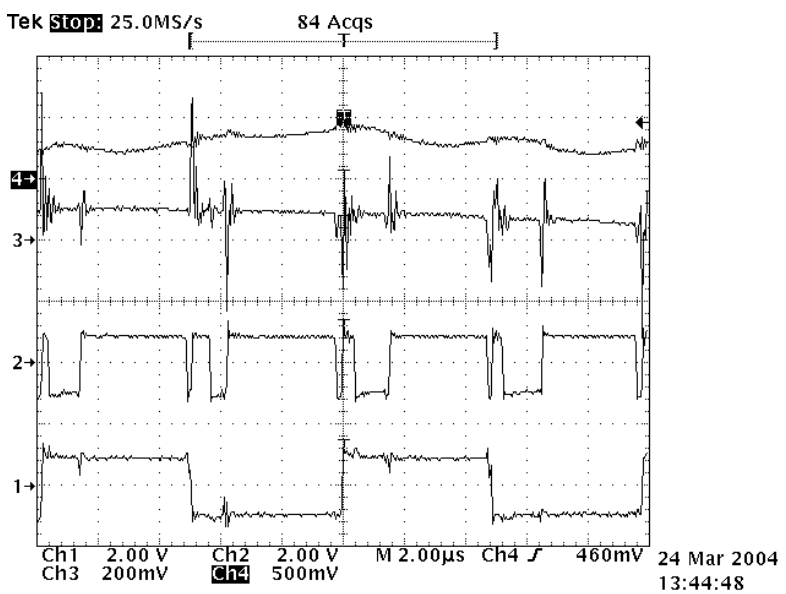

Fig. 10. Detailed SICAM operation: 1) HF-link voltage (100V/div), 2) bridge voltage $(100 \mathrm{~V} / \mathrm{div}), 3)$ voltage reference $(2 \mathrm{~V} / \mathrm{div}), 4)$ output voltage $(5 \mathrm{~V} / \mathrm{div})$ with $10 \mathrm{kHz}$ reference

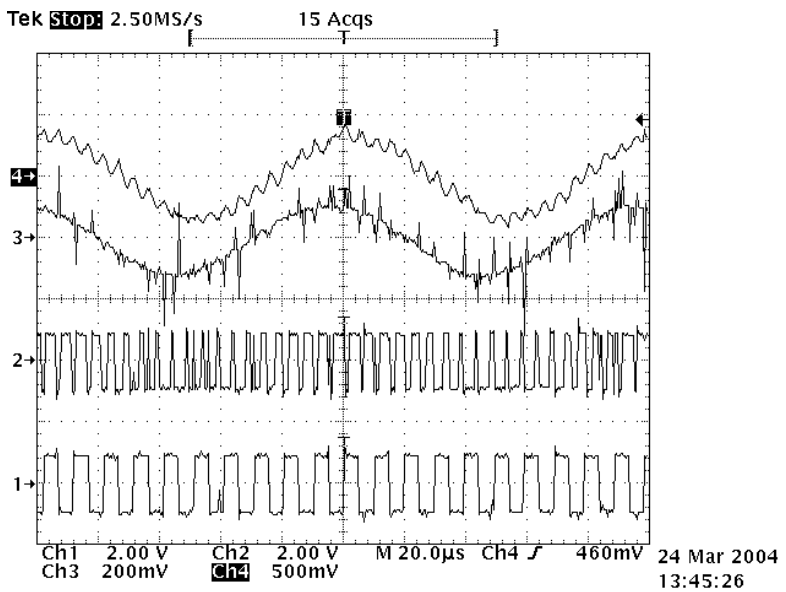

Fig. 11. SICAM operation: 1) HF-link voltage (100V/div), 2) bridge voltage $(100 \mathrm{~V} / \mathrm{div}), 3)$ voltage reference $(2 \mathrm{~V} / \mathrm{div}), 4)$ output voltage $(5 \mathrm{~V} / \mathrm{div})$ with $10 \mathrm{kHz}$ reference

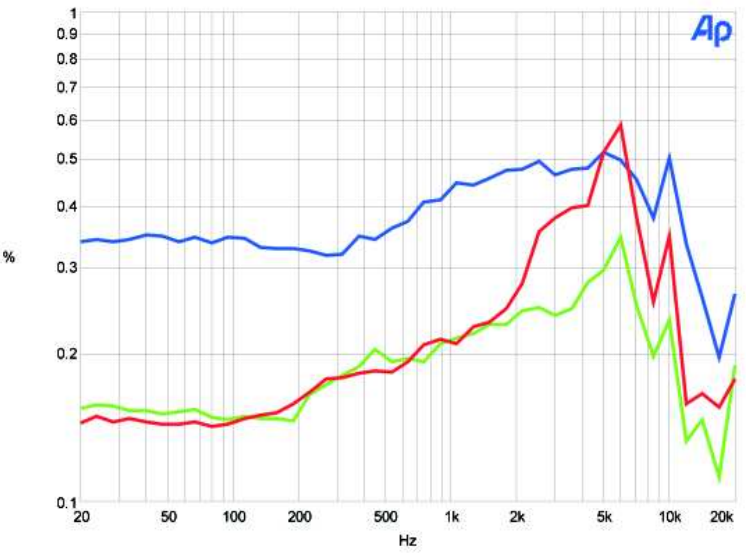

Fig. 12. THD+N versus frequency $(\mathrm{BW}=22 \mathrm{kHz}$ and $\mathrm{AES} 17$ filter): top $10 \mathrm{~W}$, bottom - $50 \mathrm{~W}$, middle $-75 \mathrm{~W}$

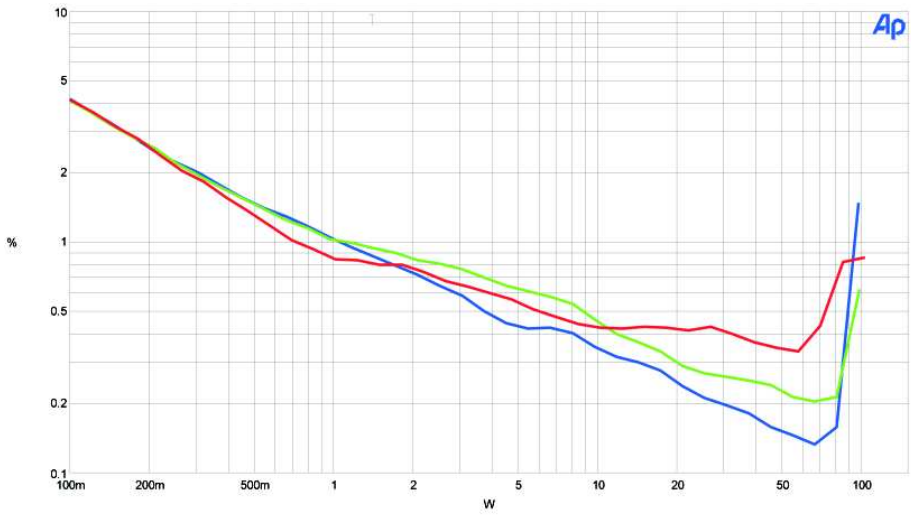

Fig. 13. THD+N versus power $(\mathrm{BW}=22 \mathrm{kHz}$ and $\mathrm{AES} 17$ filter): bottom $100 \mathrm{~Hz}$, middle $-1 \mathrm{kHz}$, top $-6.67 \mathrm{kHz}$

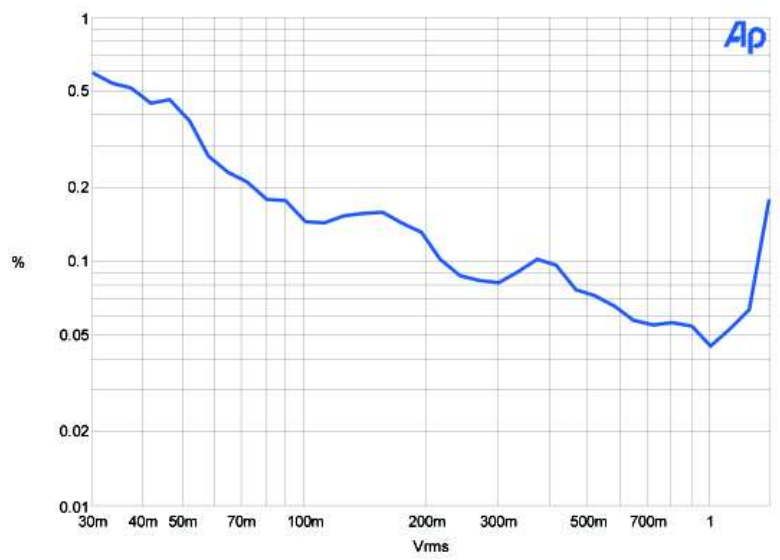

Fig. 14. Intermodulation distortion

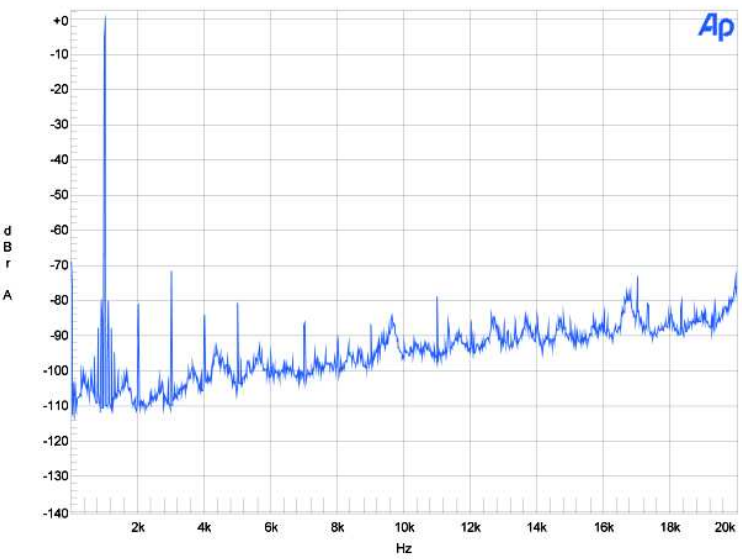

Fig. 15. FFT at $50 \mathrm{~W}$ with a signal of $1 \mathrm{kHz}$ 


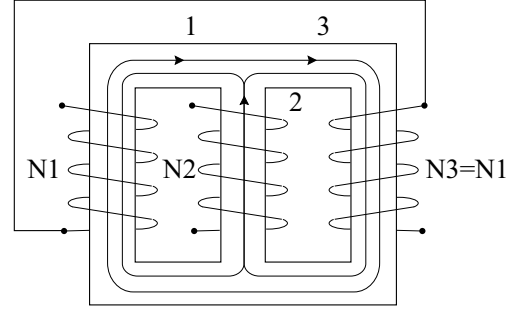

Fig. 16. Integrated magnetics

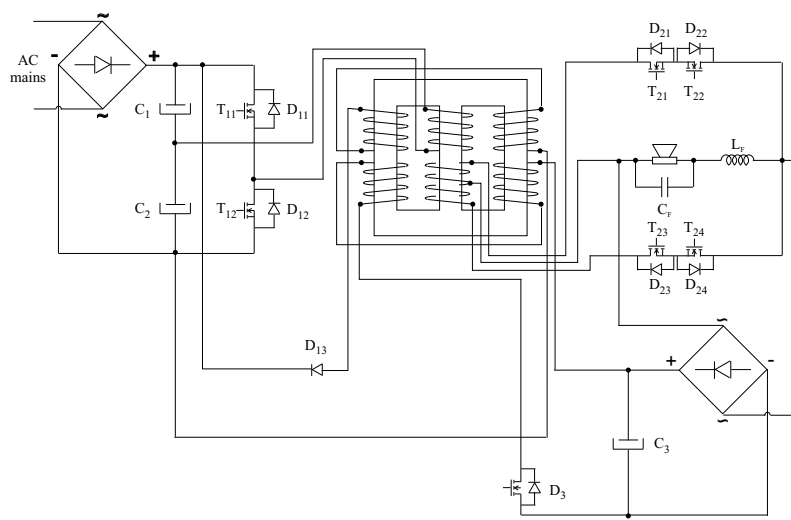

Fig. 17. Integrated magnetics for the SICAM with active clamp

magnetic structure associated with the windings $N_{1}$ and $N_{3}$ on the outer legs.

One particular implementation of the proposed integrated magnetics for the SICAM with active capacitive voltage clamp, where the auxiliary transformer uses the outer legs, is shown in Fig. 17. If the tiny air gap, being already inserted in the magnetic core for linearization purposes and better resistance to transient saturation problems, cannot store enough magnetic energy in the outer legs for the auxiliary converter, other arrangements like putting the auxiliary transformer winding on the central leg with additional air gap are also possible.

\section{CONCLUSION}

This paper discussed the possibility of using the direct energy conversion approach in audio power amplification. It was shown that the proposed integrated audio power amplifiers have a great potential, resulting in simpler and more compact designs, higher efficiency, less components and volume and yielding lower price. The load current commutation problem present in the output stage bidirectional bridge can be coped by designing active load clamps, without sacrificing efficiency and performance. The proposed SICAM with active capacitive voltage clamp has proved the viability of the direct conversion audio power amplification. The presented integrated magnetics shows an interesting way to combine the main and the auxiliary transformers on the same magnetic core and by that achieve significant savings.

\section{ACKNOWLEDGMENT}

The SICAM project is funded under the grant of the Danish Energy Authority EFP no. 1273/02-0001 and is performed in cooperation with Bang \& Olufsen ICEpower a/s in Kgs. Lyngby, Denmark.

\section{REFERENCES}

[1] K. Nielsen, "Audio power amplifier techniques with energy efficient power conversion," Ph.D. dissertation, Technical University of Denmark, Kgs. Lyngby, Denmark, April 1998.

[2] P. Ljušev and M. Andersen, "Safe-commutation principle for direct single-phase ac-ac converters for use in audio power amplification," in Proc. Nordic Workshop on Power and Industrial Electronics NORPIE 2004, Trondheim, Norway, June 14-16 2004.

[3] — , "Approaches to building single-stage ac/ac conversion switchmode audio power amplifiers," in Proc. 11th International Power Electronics and Motion Control conference EPE-PEMC 2004, Riga, Latvia, September 2-4 2004

[4] K. Nielsen and L. M. Fenger, "The active pulse modulated transducer (at) a novel audio power conversion system architecture," in 115th Convention of the Audio Engineering Society, AES Proceedings, October 10-13 2003, preprint 5866 .

[5] A. Zuckerberger, D. Weinstock, and A. Alexandrovitz, "Single-phase matrix converter," IEE Proceedings on Electric Power Applications, vol. 144, no. 4, pp. 235-240, July 1997.

[6] P. Espelage and B. Bose, "High-frequency link power conversion," IEEE Transactions on Industry Applications, vol. IA-13, no. 5, pp. 387-94, 1977.

[7] P. Ljušev and M. Andersen, "Switching-mode audio power amplifiers with direct energy conversion," in 118th Convention of the Audio Engineering Society, AES Proceedings, Barcelona, Spain, May 28-31 2005.

[8] D. Mitchell, "Dc to low frequency inverter with pulse width modulated high frequency link," U.S. patent 4,339,791, July 1982.

[9] D. Gurwitz and L. J. Berman, "Static inverter," UK Patent Application: GB2087171A, May 1982.

[10] P. Ljušev and M. Andersen, "Self-oscillating modulators for direct energy conversion audio power amplifiers," in Proc. 27th International AES Conference on Efficient Audio Power Amplification (submitted), Hillerd, Denmark, September 2-4 2005.

[11] B. E. Attwood, L. E. Hand, and L. C. Santillano, "Audio amplifier with phase modulated pulse width modulation," U.S. patent 4,992,751, February 1991

[12] R. W. Erickson and D. Maksimović, Fundamentals of Power Electronics. Kluwer Academic Publishers, 2001, second edition.

[13] P. Ljušev and M. Andersen, "New active load voltage clamp for hflink converters," in Proc. The 40th International Universities Power Engineering Conference UPEC 2005, Cork, Ireland, September 7-9 2005. 